\title{
HOSPEDEIROS E CICLOS SUCESSIVOS DE MULTIPLICAÇÃO AFETAM A DETECÇÃO DE FUNGOS MICORRÍZICOS ARBUSCULARES EM ÁREAS IMPACTADAS POR MINERAÇÃO GESSEIRA ${ }^{1}$
}

\author{
Adália Cavalcanti do Espírito Santo Mergulhão², Márcia do Vale Barreto Figueiredo³, Hélio Almeida
} Burity $^{3}$ e Leonor Costa Maia ${ }^{4}$

\begin{abstract}
RESUMO - O objetivo deste trabalho foi verificar a influência de diferentes plantas hospedeiras e de ciclos de multiplicação em potes de cultura sobre a detecção e avaliação da diversidade de fungos micorrízicos arbusculares (FMA) no semiárido, em áreas de caatinga preservadas e impactadas por mineração de gesso no semiárido (Araripina, PE). Foram selecionadas quatro áreas de coleta: AN - caatinga nativa preservada, AM - arredores da mina, AR-rejeito e AI - interface entre o depósito de rejeito e uma área de caatinga degradada pela mineração, todas com solo do tipo Latossolo Amarelo, variando de franco-arenoso a argiloso, com pH de 5,7 a 7,5; 8 a $161 \mathrm{mg} \cdot \mathrm{dm}^{-3} \mathrm{P}$ e 0,23 a $79,8 \mathrm{mg} \cdot \mathrm{dm}^{-3} \mathrm{Fe}$. Amostras foram retiradas desses solos para exame imediato e preparo de culturas-armadilha para FMA, utilizando-se como plantas hospedeiras amendoim (Arachis hypogaea L.) e sorgo granífero (Sorghum bicolor (L.) Moench) cultivar IPA-7301011. Logo após a coleta e depois de cada ciclo de multiplicação na cultura-armadilha (três ciclos de três meses cada), foram avaliados em amostras de raízes e de solo rizosférico: colonização radicular, número de esporos, diversidade e similaridade de espécies de FMAs entre as áreas. As plantas de amendoim apresentaram valores mais altos de colonização radicular $(76,5$ a $99,5 \%)$ que as de sorgo ( $21 \%$ a $73 \%)$. Maior produção de esporos ocorreu no $2^{\circ}$ e no $3^{\circ}$ ciclo de culturasarmadilha. Foram identificadas 25 espécies de FMAs nas áreas estudadas, onde se destacaram Glomus intraradices Schenck \& Smith, Glomus mosseae Gerdemann \& Trappe e Paraglomus occultum Morton \& Redecker, pela alta densidade de esporos produzidos. As áreas apresentaram índice de similaridade de espécies de FMAs variando de 40 a $67 \%$, com os maiores índices entre as áreas impactadas.
\end{abstract}

Palavras-chave: Culturas-armadilha, Arachis hypogaea, Sorghum bicolor e recuperação de áreas alterada.

\section{HOST AND SUCCESSIVE CYCLES OF MULTIPLICATION AFFECT THE DETECTION OF ARBUSCULAR MYCORRHIZAL FUNGI IN GYPSUM MINING IMPACTED AREAS}

\begin{abstract}
The objective of this report was to verify the influence of host plants and successive multiplication cycles on pot cultures for detection of the diversity of arbuscular mycorrhizal fungi (AMF) in the semiarid, in area with native, preserved "caatinga" and areas impacted by gypsum mining in Araripina, PE. Four areas were selected: AN - "caatinga" native, preserved caatinga; AM - surroundings of the mine; AR - waste and AI - interface between the waste deposit area and an area degraded by mining, all of them with Yellow Latosol, varying from franc sandy to clay, with $\mathrm{pH} 5.7$ to $7.5 ; 8$ to $161 \mathrm{mg} . \mathrm{dm}^{-3} \mathrm{P}$ and 0.23 to $79.8 \mathrm{mg} . \mathrm{dm}^{-}$ ${ }^{3} \mathrm{Fe}$. Soil samples were taken for exam and preparation of AMF trap cultures, using peanut (Arachis hypogaea L.) and sorghum (Sorghum bicolor (L.) Moench cv IPA-7301011) as hosts. Soon after the collection and after each multiplication cycle in trap cultures ( 3 cycles/3 months each), root colonization, spore number,
\end{abstract}

\footnotetext{
${ }^{1}$ Recebido em 18.09.2007 e aceito para publicação em 06.03.2009.

${ }^{2}$ Laboratório de Genômica do Instituto Agronômico de Pernambuco (IPA). E-mail: <adalia@ipa.br>.

${ }^{3}$ Laboratório de Biologia de Solo da Empresa Pernambucana de Pesquisa Agropecuária. E-mail: <mbarreto@elogica.com.br>.

${ }^{4}$ Departamento de Micologia da Universidade Federal de Pernambuco (UFPE). E-mail: <leonorcmaia@ yahoo.com.br>.
} 
diversity and similarity of species were evaluated in soil and root samples. The peanut plants presented higher values of root colonization ( 76.5 to $99.5 \%$ ) than those of sorghum (21\% to 73\%). Higher spore production occurred in the second and third multiplication cycles. Twenty-five AMF species were identified in the areas, with Glomus intraradices Schenck \& Smith, Glomus mosseae Gerdemann \& Trappe, and Paraglomus occultum Morton \& Redecker producing higher spore density than the other species identified. The index of similarity of AMF species varied from 40 to $67 \%$, with the highest values found in the impacted areas.

Keywords:Trap-culture, Arachis hypogaea, Sorghum bicolor and altered site recovery.

\section{INTRODUÇÃO}

Os fungos micorrízicos arbusculares (FMA), importantes componentes da comunidade microbiana do solo, formam associação simbiótica com o sistema radicular da maioria das plantas terrestres, participando do processo de sucessão vegetal e contribuindo para a diversificação e estabilidade dos ecossistemas naturais (VAN DER HEIDJEN et al., 1998). Aassociação micorrízica é crítica para o estabelecimento, desenvolvimento e manutenção desses ecossistemas e para a recuperação daqueles severamente perturbados (REEVES e REDENTE, 1991), pois a planta micorrizada está mais apta a colonizar áreas degradadas, uma vez que apresenta estratégia nutricional superior à da planta não colonizada (SOUZA e SILVA, 1996).

A ocorrência de FMA é determinada por meio de avaliações quantitativas, que envolvem procedimentos como colonização radicular e densidade de esporos no solo; e qualitativas, em que a presença de diferentes fungos é determinada para gênero e, ou, espécie (SIQUEIRA e KLAUBERG FILHO, 2000). Stutz e Morton (1996) demonstraram que culturas-armadilha podem ser usadas para estimar a distribuição das espécies de FMA e sua equitabilidade, e o uso de culturasarmadilha sucessivas fornece visão mais ampla da diversidade de espécies em ambientes com alta proporção de FMA não esporulantes. Ainda segundo esses autores, essa estratégia possibilita melhor avaliação das espécies indígenas, principalmente em solos de zonas áridas e semiáridas.

Apesar de reconhecidamente importantes, dados ecológicos e biológicos básicos sobre os FMAs ainda são necessários para melhor entendimento da associação e dos seus benefícios (FITTER, 1990). Nesse contexto e considerando que trabalhos sobre a ocorrência de FMAs em ecossistemas naturais ou impactados são escassos, justifica-se a importância de pesquisas nessa área.

R. Árvore, Viçosa-MG, v.33, n.2, p.227-236, 2009
A maior parte da região semiárida brasileira é coberta pelo bioma Caatinga, que se estende por mais de 800.000 $\mathrm{km}^{2}$, em nove Estados, ocupando $70 \%$ do Nordeste e $11 \%$ do território nacional. Esse bioma, exclusivamente brasileiro, é hoje reconhecido pela riqueza em espécies animais e vegetais. Com relação aos fungos, incluindo os FMAs, trabalhos recentes mostraram que também há grande diversidade (GUSMÃO e MAIA, 2006). No entanto, considerando a grande extensão do semiárido, é necessária a ampliação do conhecimento sobre a diversidade para melhor compreensão da relação plantafungo micorrízico nesse ambiente tão particular, que em algumas áreas se encontra degradado por ações antrópicas, entre as quais a atividade mineradora. Nessas áreas, o processo de remoção da vegetação e a perda da camada superficial orgânica, com a consequente modificação da estrutura do solo, eliminam os propágulos de FMAs, provocando drástica redução na capacidade infectiva dos fungos, o que pode afetar a sucessão das plantas pela limitação de espécies vegetais capazes de crescer sem estabelecer a simbiose micorrízica. Foi comprovado que a utilização de FMAs na recuperação de áreas degradadas proporciona aos vegetais maior rapidez no crescimento e produção de biomassa (SOUZA e SILVA, 1996).

Trabalhos realizados sobre FMA em áreas preservadas e exploradas mostram a importância desses fungos nos ambientes estudados (CAPRONI et al., 2003, 2005; MARINHO et al., 2004; MELLONI et al., 2003; MERGULHÃO et al., 2007; SILVA et al., 2001, 2005). Em caatinga nativa, na região de Xingó, AL, Souza et al. (2003) identificaram 24 táxons de FMAs, com maior representatividade das famílias Acaulosporaceae e Glomaceae. Esses autores também observaram que os fungos formavam associação com a maioria das espécies vegetais encontradas, apesar das limitações climáticas da região. Estudando a composição das comunidades de FMAs em áreas revegetadas com Acacia mangium após a mineração 
de bauxita na região de Porto Trombetas, no Pará, Caproni et al. (2005) verificaram que Glomus clarum Nicolson \& Schenck e Gigaspora margarita Becker \& Hall apresentavam características distintas de esporulação, indicando adaptações aos primeiros estágios de recuperação nas áreas degradadas pela mineração. Em área de caatinga preservada e em área degradada por mineração de cobre, na Bahia, Silva et al. (2005) identificaram 15 espécies de FMAs e observaram, nas áreas impactadas, forte redução na diversidade vegetal e na comunidade de espécies de FMAs, afetada quantitativa e qualitativamente pela atividade mineradora. Em áreas salinizadas no Vale do São Francisco, em PE, Yano-Melo et al. (2003) identificaram 21 táxons de FMAs, com destaque para Glomus mosseae Gerdemann \& Trappe e Glomus intraradices Schenck \& Smith e, segundo os citados autores, o primeiro ciclo de multiplicação em cultura-armadilha favoreceu a esporulação dos FMAs, que decresceu a partir do segundo ciclo.

Conhecer a diversidade de espécies de FMAs em áreas impactadas também é importante, considerandose que os dados podem servir para orientar os possíveis trabalhos de recuperação a serem realizados. Assim, este estudo teve como objetivo verificar a influência do fotobionte (amendoim e sorgo) e de sucessivos ciclos de multiplicação em potes de cultura sobre a detecção de FMA em solos de caatinga nativa preservada e em áreas impactadas por mineração de gesso, bem como observar a similaridade de FMA entre as áreas estudadas, para melhor conhecimento de aspectos relacionados à ocorrência e distribuição desses fungos simbiontes no semiárido.

\section{MATERIAL E MÉTODOS}

Foram selecionadas áreas de caatinga nativa e impactada por mineração de gipsita no Município de Araripina, PE (7॰ 29' 00” S, 40³6’00” W). A região apresenta clima semiárido mesotérmico e a vegetação predominante é caatinga hiperxerófila sobre Latossolo Amarelo (CAVALCANTI e LOPES, 1994). Nas áreas estudadas, foram identificadas 42 espécies, representando 25 famílias de fanerógamas, sendo as mais bem representadas: Leguminosae (Mimosoidae), com seis espécies: Parapiptadenia zehntneri (Harms) M.P., Mimosa tenuiflora (Willd) Poir., Mimosa ophthalmocentra Mart. Bemth., Anadenanthera colubrina (Vell.) Brenan., Prosopis juliflora DC.,
Desmanthus virgatus (L.) Willd. e Euphorbiaceae, com quatro espécies: Croton sonderianus Mull. Arg., Croton sp., Manihot sp. e Jatropha mollissima (Pohl.) Baill.

A coleta de solo foi realizada em quatro áreas: (a) caatinga nativa preservada (AN); controle; (b) arredores da mina (AM); (c) rejeito (AR); e (d) interface entre o depósito de rejeito e uma área de caatinga degradada pela mineração (AI). Em cada área foram coletadas 10 amostras de solo (5-20 cm de profundidade), preferencialmente na rizosfera de plantas, sendo os pontos definidos aleatoriamente. Parte do solo foi encaminhada para o Instituto Agronômico de Pernambuco (IPA) para análises física, química e de fertilidade do solo (Tabela 1).

Foram preparadas culturas-armadilha para FMA, com o solo coletado das áreas estudadas. O solo foi diluído em areia (2:1) e colocado em copos plásticos com capacidade para $500 \mathrm{~mL}$, tendo como plantas hospedeiras o amendoim (Arachis hypogaea L.) e o sorgo granífero (Sorghum bicolor (L.) Moench) cultivar IPA-7301011. Foram mantidos três ciclos dessas culturas em casa de vegetação, cada um por cerca de três meses; durante esse período, as plantas foram regadas em dias alternados. A temperatura e a umidade relativa do ar foram medidas diariamente, variando de 21 a 35 ${ }^{\circ} \mathrm{C}$ e de 31 a $88 \%$, respectivamente.

As avaliações da densidade de esporos e colonização radicular foram feitas antes e depois de cada ciclo de multiplicação dos FMA em culturasarmadilha. Os esporos foram extraídos do solo por peneiramentoúmido (GERDEMANN e NICOLSON, 1963) e centrifugação em água e sacarose 50\% (JENKINS, 1964) e observados ao microscópio. Os esporos foram montados em lâminas com PVLG ou reagente de Melzer + PVLG (1:1). Para identificação taxonômica foram consultados Schenck e Pérez (1990) e a home page http://invam.caf.wvu.edu. As raízes foram preparadas de acordo com o método de clarificação (KOH 10\%) e coloração com $0,05 \%$ de azul Trypan em lactoglicerol (PHILLIPS e HAYMAN, 1970). Foram avaliados 200 fragmentos de raízes com cerca de $1 \mathrm{~cm}$ e considerado colonizado aquele que continha arbúsculos, esporos e micélio, além de vesículas e células auxiliares.

A similaridade de FMA entre as áreas foi avaliada pelo Índice de Sörensen (BROWER e ZAR, 1984), pela fórmula $S=2 c / a+b x 100$, em que c = número de espécies comuns às duas áreas ( 1 e 2), a = número de espécies na área 1 e b = número de espécies na área 2 .

R. Árvore, Viçosa-MG, v.33, n.2, p.227-236, 2009 


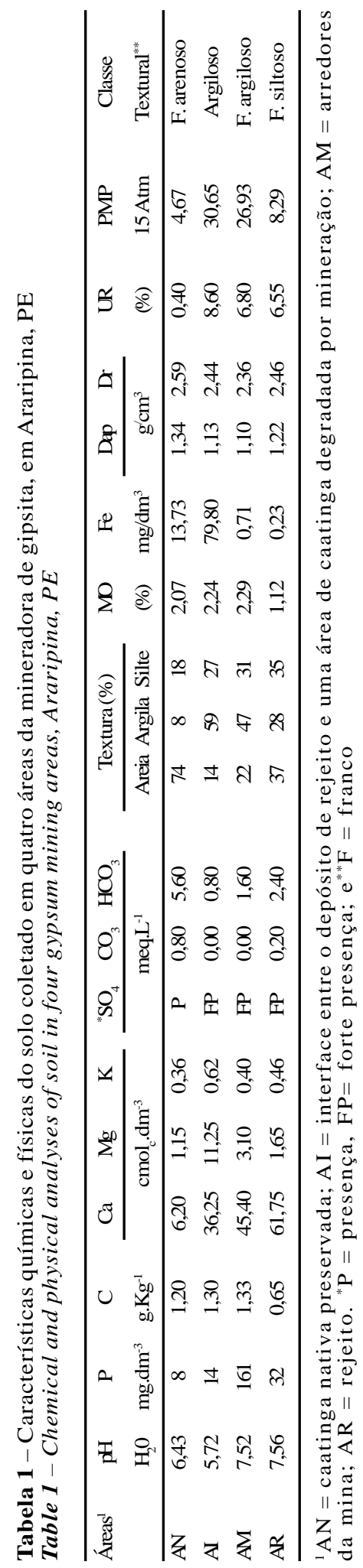

Para avaliar as diferenças considerando as áreas (AN, AM, AI e AR) e ciclos de multiplicação (inicial, $1^{\circ}, 2^{\circ}$ e $3^{\circ}$ ), bem como a interação entre esses fatores, usou-se a análise de variância (ANOVA). O número de esporos foi transformado em $\log (X+1,5)$, a colonização radicular em $\sqrt{ }(X+0,5)$ e as médias comparadas pelo teste de Tukey $(\mathrm{P} \leq 0,05)$, utilizandose o programa Sanest (ZONTA et al., 1984).

\section{RESULTADOS E DISCUSSÃO}

Os solos estudados (Tabela 1) apresentaram, conforme Tomé Júnior (1997), acidez da solução aquosa de média a fraca; teores de fósforo baixos na área de caatinga nativa preservada, médios nas áreas AI e AR e alto na área AM; cálcio e potássio variando de médio a alto e magnésio, atingindo alto teor na área de interface. Doze espécies de FMAs foram registradas na área nativa preservada, em que o pH estava próximo da neutralidade $(6,43)$ e os níveis de $\mathrm{P}, \mathrm{Ca}$ e $\mathrm{Mg}$ menores do que nas outras áreas (AI, AM e AR). Acaulospora mellea Spain \& Schenck, Glomus etunicatum Becker \& Gerdemann, Glomus intraradices Schenck \& Smith, Glomus mosseae Gerdemann \& Trappe e Paraglomus occultum Morton \& Redecker parecem ser mais resistentes a valores mais elevados de $\mathrm{pH}, \mathrm{P}, \mathrm{Ca}, \mathrm{Mg}$ e $\mathrm{SO}_{4}$, sendo encontradas em todas as áreas estudadas (Tabelas 1 e 2). Archaeospora leptoticha Morton \& Redecker, Scutellospora auriglobosa Walker \& Sanders, Scutellospora calospora Walker \& Sanders e Scutellospora heterogama Walker \& Sanders não foram registradas nas áreas impactadas (AI, AM e AR) e Acaulospora foveata Trappe \& Janos, Acaulospora longula Spain \& Schenck, Gigaspora margarita Becker \& Hall e Scutellospora heterogama só foram detectadas nas culturas-armadilha, não sendo observadas em nenhuma das amostras recém-coletadas (Tabela 2).

Vinte e cinco táxons de FMA foram encontrados nas áreas estudadas após sucessivos ciclos de cultura em pote, sendo mais representados por número de espécies: Glomus (10 espécies), Acaulospora (7) e Scutellospora (4) (Tabela 2). Resultados similares foram obtidos por outros autores em região semiárida (YANO-MELO et al., 2003; CAPRONI et al., 2005; SILVA et al., 2005). Segundo Allen et al. (1995), algumas espécies de Glomus, Acaulospora e Scutellospora possuem ampla distribuição geográfica. Estudando os efeitos do uso de diferentes plantas hospedeiras na detecção da diversidade de FMA, Carrenho et al. (2002) verificaram que amendoim e milho propiciavam oestabelecimento de membros de Acaulosporaceae, enquanto o sorgo favorecia o registro de espécies de Glomaceae. 
Tabela 2 - Número de esporos $\left(50 \mathrm{~g}^{-1}\right.$ solo) obtidos na coleta inicial e após sucessivos ciclos de cultura em pote (com sorgo e amendoim) e em solos das áreas de caatinga nativa preservada e impactada por mineração de gipsita

Table 2 - Espore number (50g-1 soil) after successive cycles of pot culture (with sorghum and groundnut) and after harvest (initial) in soil of the areas of "caatinga" preserved and impacted by gypsum mining

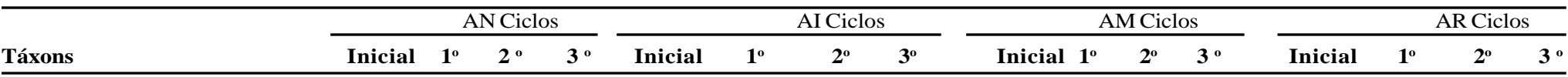

Acaulospora excavata

Inicial $\mathbf{1}^{\mathrm{o}} \mathbf{2}^{\circ}-3^{\circ}$

Inicial

Inicial $\mathbf{1}^{\circ} \quad 2^{\circ} \quad 3^{\circ}$

nicial

$\mathbf{2}^{\mathbf{o}}$

Ingleby \& Walker

A. foveata Trappe \& Janos

A. longula Spain \& Schenck

A. mellea Spain \& Schenck

A. rehmii Sieverding \& Toro

A. scrobiculata Trappe

A. tuberculata Janos \& Trappe

Archaeospora leptoticha

Morton \& Redecker

Entrophospora infrequens

Ames \& Schneider

Gigaspora gigantea Nicol. \& Gerd.

Gigaspora margarita

Becker \& Hall

\% Glomus clavisporum

G. etunicatum Becker \& Gerd.

G. fasciculatum Gerd. \& Trappe

G. halonatum Rose \& Trappe

G. intraradices Schenck \& Smith

G. macrocarpum Tulasne

G. mosseae Nicol. \& Gerd.

G. pallidum Hall

G. sinuosum Almeida \& Schenck

Paraglomus occultum

Morton \& Redecker

Scutellospora auriglobosa Hall

S. calospora Nicol. \& Gerd.

S. heterogama Nicol. \& Gerd.

S. pellucida Nicol \& Schenck

Total de Táxons

$\begin{array}{llllllll}12 & 4 & 9 & 4 & 6 & 7 & 3 & 5\end{array}$

$\begin{array}{llll}5 & 2 & 4 & 5\end{array}$

$\begin{array}{llll}2 & 5 & 5 & 4\end{array}$

气ิ $\mathrm{AN}=$ caatinga nativa preservada; $\mathrm{AI}=$ interface entre o depósito de rejeito e uma área de caatinga degradada por mineração; $\mathrm{AM}=$ arredores da mina; $\mathrm{e} \mathrm{AR}=$ rejeito. 
O uso de culturas-armadilha e ciclos sucessivos favoreceu a multiplicação de esporos de Acaulospora scrobiculata Trappe, Glomus intraradices, Glomus mosseae e Paraglomus occultum (Tabela 2). Do mesmo modo, os ciclos sucessivos também favoreceram a colonização radicular nas plantas de sorgo e amendoim (Tabela 3). A utilização desses hospedeiros favoreceu a esporulação de espécies com germinação tardia e o sorgo, além de suportar condições adversas do solo, apresenta ciclo de vida mais longo que o amendoim. As duas espécies de plantas hospedeiras são micotróficas facultativas (SIEVERDING, 1991), ou seja, necessitam em diferentes graus da associação micorrízica, dependendo da fertilidade do solo. As menores taxas de colonização radicular verificadas nas plantas de sorgo, quando comparadas com as plantas de amendoim (Tabela 3), podem ser explicadas pelo fato de essa gramínea, por apresentar maior densidade, extensão e capacidade de ramificação das raízes (ROBERTSON et al., 1980), aumentar as oportunidades de formação de pontos de infecção, aproximando as raízes a uma maior quantidade de esporos, ao longo do substrato. Com mais área radicular a ser explorada, os pontos de infecção e a posterior colonização podem ter sido "diluídos".

O porcentual de colonização inicial das raízes coletadas (obtido antes dos ciclos de multiplicação) foi maior nas áreas de maior impacto ambiental (AM e AR), diferindo significativamente das demais. Houve interação entre os fatores estudados (áreas e ciclos de multiplicação) para o percentual de colonização radicular de FMA em plantas de sorgo $(\mathrm{P}<0,004) \mathrm{e}$ amendoim $(\mathrm{P}<0,001)$. Após sucessivos ciclos de multiplicação, a colonização radicular em plantas de amendoim variou de 76,5 a 99,5\%. Com relação ao sorgo, essa variação foi de 21 a $73 \%$ (Tabela 3 ). As plantas de amendoim apresentaram os valores mais altos de colonização radicular. Resultado similar foi obtido por Carrenho et al. (2002). Os níveis mais elevados de colonização radicular, verificados em amendoim, podem ser devidos à composição diferenciada dos exsudatos radiculares. Redmond et al. (1986) demonstraram que algumas flavonas são produzidas exclusivamente por leguminosas, e D'arcy-Lameta (1988) observou que diferentes leguminosas produzem diversos conjuntos de compostos flavonóides. Esses compostos podem ter favorecido a ocupação das raízes pelos FMAs, por influenciar o crescimento e direcionamento das hifas dos esporos em germinação, similarmente ao efeito indutor da luteolina sobre a nodulação de Rhizobium (PETERS et al., 1986). No entanto, a presença de FMA nas raízes de leguminosas também contribui para expandir a área de captação dos nutrientes que chegam até as raízes pelo processo de difusão (JESUS et al., 2005). Alguns produtos de excreção radicular têm sido verificados estimulando o crescimento e a ramificação do micélio formado a partir da germinação dos esporos de FMA (GIANINAZI-PEARSON et al., 1989).

Observou-se maior esporulação na área preservada, que apresentou diferenças significativas desse parâmetro quando comparada com as demais áreas estudadas. O número de esporos logo após a coleta variou de um a quatro esporos $\mathrm{g}^{-1}$ solo, e a montagem de mais de um ciclo de culturas em pote, com os solos coletados, favoreceu a esporulação dos FMAs (Figuras 1 e 2).

Tabela 3 - Colonização radicular de fungos micorrízicos arbusculares em sorgo e amendoim após sucessivos ciclos de multiplicação em casa de vegetação

Table 3 - Radicular colonization of arbuscular mycorrhizal fungi in Sorghum and groundnut after successive cycles of multiplication in greenhouse

\begin{tabular}{|c|c|c|c|c|c|c|}
\hline \multirow[b]{3}{*}{ Áreas $^{2}$} & & \multicolumn{5}{|c|}{ Percentagem de Colonização Radicular $(\%)^{1}$} \\
\hline & \multicolumn{3}{|c|}{ Sorgo* } & \multicolumn{3}{|c|}{ Amendoim ${ }^{* *}$} \\
\hline & $1^{\circ} \mathrm{Ciclo}$ & $2^{\circ} \mathrm{Ciclo}$ & $3^{\circ} \mathrm{Ciclo}$ & $1^{\circ} \mathrm{Ciclo}$ & $2^{\circ}$ Ciclo & $3^{\circ} \mathrm{Ciclo}$ \\
\hline $\mathrm{AN}$ & $37,0 \mathrm{bB}$ & $68,0 \mathrm{aA}$ & 73,0 aA & 99,5 aA & $96,0 \mathrm{aA}$ & 97,5 aA \\
\hline AI & $38,5 \mathrm{bB}$ & $45,0 \mathrm{bAB}$ & $53,0 \mathrm{bA}$ & $76,5 \mathrm{cB}$ & $98,0 \mathrm{aA}$ & 96,0 aA \\
\hline AM & $21,0 \mathrm{cB}$ & $51,0 \mathrm{bA}$ & $62,0 \mathrm{abA}$ & $86,0 \mathrm{bB}$ & $95,0 \mathrm{aA}$ & 96,5 aA \\
\hline AR & 63,0 aA & $60,5 \mathrm{abA}$ & $67,0 \mathrm{abA}$ & 98,0 aA & 99,0 aA & $98,0 \mathrm{aA}$ \\
\hline${ }^{*} \mathrm{CV}(\%)=13$ & ${ }^{* *} \mathrm{CV}(\%)=3$ & & & & & \\
\hline
\end{tabular}

${ }^{1}$ Médias seguidas da mesma letra minúscula na mesma coluna ou maiúscula na mesma linha não diferem significativamente pelo teste de Tukey $(\mathrm{P}<0,05)$. Médias originais, porém para a análise de variância, foram transformadas em $\mathrm{v}(\mathrm{X}+0,5) .{ }^{2} \mathrm{AN}=$ caatinga nativa preservada; $\mathrm{AI}=$ interface entre o depósito de rejeito e uma área de caatinga degradada por mineração; AM = arredores da mina; e AR = rejeito

R. Árvore, Viçosa-MG, v.33, n.2, p.227-236, 2009 
Tabela 4 - Porcentual de similaridade (Sörensen) de FMA entre as áreas estudadas no Município de Araripina, $\mathrm{PE}$

Table 4-Sörensen's index of similarity of AMF among the studied areas in Araripina, $P E$

\begin{tabular}{lcccc}
\hline & \multicolumn{4}{c}{ Similaridade de FMA (\%) } \\
\cline { 2 - 5 } Áreas $^{1}$ & AN & AI & AM & AR \\
\hline AN & 100 & 40 & 57 & 44 \\
AI & & 100 & 67 & 59 \\
AM & & & 100 & 67 \\
AR & & & & 100 \\
\hline
\end{tabular}

${ }^{1} \mathrm{AN}=$ caatinga nativa preservada; $\mathrm{AI}=$ interface entre o depósito de rejeito e uma área de caatinga degradada por mineração; $\mathrm{AM}=$ arredores da mina; e AR = rejeito

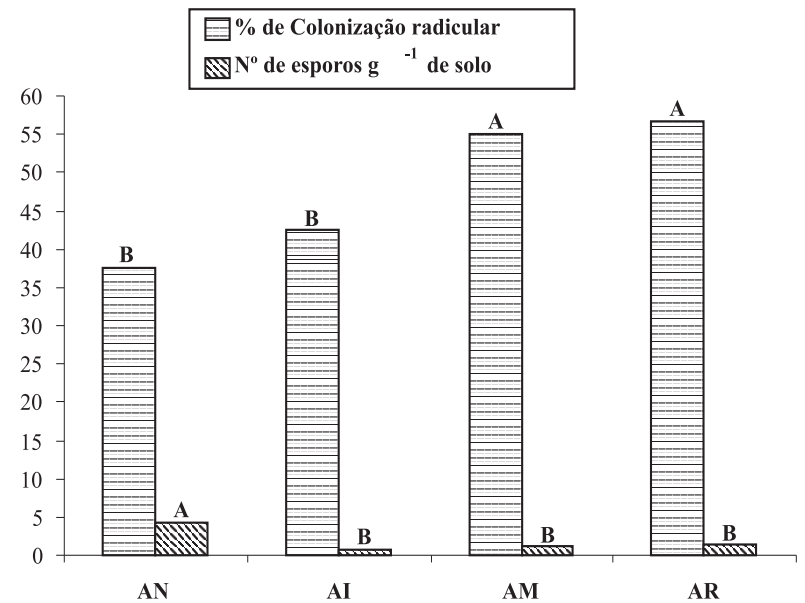

Figura 1 - Colonização radicular e número de esporos de fungos micorrízicos arbusculares obtidos sem ciclos de multiplicação em solos de caatinga nativa e impactados por mineração de gipsita. Médias seguidas da mesma letra maiúscula não diferem significativamente entre si, pelo teste de Tukey $(\mathrm{P}<0,05)$. Médias originais, porém para a análise de variância, a \% de colonização radicular foram transformadas em $\sqrt{ }(X+0,5)$ e o número de esporo, em $\log (\mathrm{X}+1,5)$. $\mathrm{AN}=$ caatinga nativa preservada; $\mathrm{AM}=$ arredores da mina; $\mathrm{AR}=$ rejeito; $\mathrm{e} \mathrm{AI}=$ interface entre o depósito de rejeito e uma área de caatinga degradada por mineração.

Figure 1-Radicular colonization and spore number of arbuscular mycorrhizal fungi without multiplication cycles in soil sample of the "caatinga" native and impacted by gypsum mining. The means followed by the same letter do not differ statistically $(P<0,05)$ from each other according to Tukey's test. Values changes $\sqrt{ }(X+0,5)$ to $\%$ radicular colonization. Values changes $\log (X+1,5)$ to spore number. $A N=$ preserved native "caatinga"; $A M=$ surroundings of the mine; $A R=$ waste $; A I=$ interface between the waste deposit area and degrading "caatinga" area.

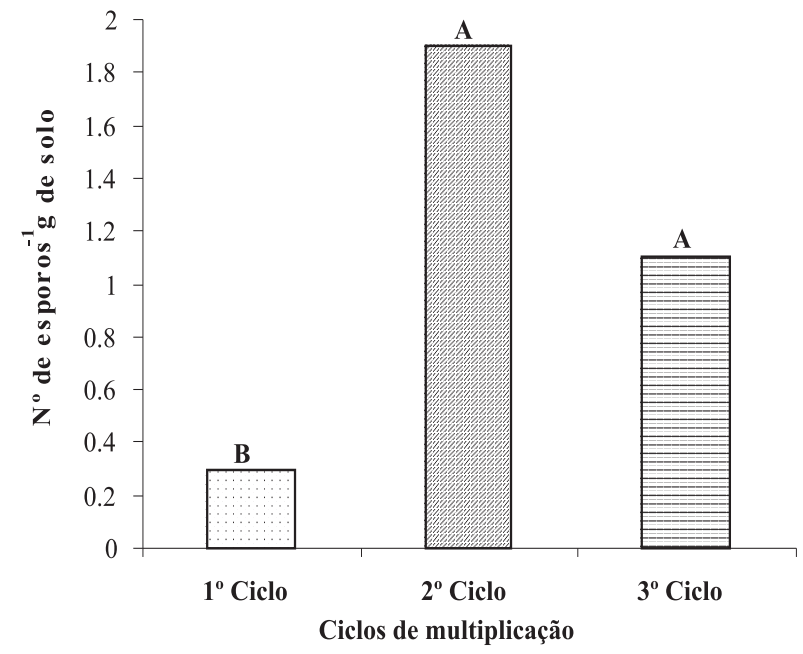

Figura 2 - Efeitos dos ciclos de multiplicação no número de esporos de fungos micorrízicos arbusculares obtidos de pote de cultura com sorgo e amendoim em casa de vegetação, independentemente da área de coleta. Médias seguidas da mesma letra maiúscula não diferem significativamente entre si, pelo teste de Tukey $(\mathrm{P}<0,05)$. Médias originais, porém para a análise de variância foram transformadas em $\log (\mathrm{X}+2)$.

Figure 2-Effects multiplication cycles in the fungi in the Sorghum and Groundnut culture pot in greenhouse, independent of harvest area. The means followed by the same letter do not differ statistically $(P<0,05)$ from each other according to Tukey's test. Values changes $\log (X+2)$.

O aumento da esporulação observado no $2^{\circ}$ e $3^{\circ}$ ciclos (Figura 2) indicou que houve acréscimo na quantidade de propágulos e as condições de multiplicação foram adequadas, sugerindo que nesses solos estudados, dependendo do grau de degradação, dois ou três ciclos são suficientes para detectar a maioria das espécies de FMAs (Tabela 2). Esses dados estão de acordo com os obtidos por Oehl et al. (2004) e Stutz e Morton (1996), os quais observaram maior produção de esporos no $2^{\circ}$ e $3^{\circ}$ ciclos, respectivamente, a partir de culturas-armadilha. Entretanto, diferem do encontrado por Yano-Melo et al. (2003), que observaram maior produção de esporos, principalmente, no $1^{\circ}$ ciclo de multiplicação. Embora todos esses solos sejam de regiões áridas e semiáridas, eles concorrem para obtenção de respostas diferenciadas: as condições edáficas, os hospedeiros e, mesmo, os FMAs presentes nesses solos. Segundo Stutz e Morton (1996), nas regiões áridas a pouca umidade é o principal fator limitante, ocasionando ausência ou baixa esporulação dos FMAs.

R. Árvore, Viçosa-MG, v.33, n.2, p.227-236, 2009 
A área de caatinga nativa preservada (AN), com menor semelhança de espécies de FMAs em relação às demais, tem solo que difere em granulometria, $\mathrm{pH}$, fósforo, cálcio, magnésio, potássio e sulfato. Observou-se elevada similaridade (67\%) de espécies de FMAs entre as áreas AI e AM e entre AM e AR (Tabela 4). Áreas impactadas em processo de recuperação apresentam estádios de sucessão caracterizados pelas modificações na composição vegetal (SOUZAe SILVA, 1996; YANO-MELO et al., 2003). Assim, a similaridade entre as áreas degradadas por atividade gesseira (AI, AM e AR) pode representar estádios de sucessão. É possível que essas áreas estejam em fase de recuperação, traduzida pela maior similaridade de espécies de FMAs, quando comparadas com o encontrado na área de caatinga nativa preservada (AN).

\section{CONCLUSÕES}

Diante dos resultados, conclui-se que:

- O uso de mais de uma planta hospedeira nas culturas-armadilha permite a identificação de maior número de espécies de FMAs e aumenta as possibilidades de associação e, consequentemente, maior esporulação.

- Culturas-armadilha contribuem para incremento da detecção e registro da ocorrência de FMA em ambientes nativos e impactados.

- Dependendo do nível de impacto do solo, são necessários dois ou três ciclos de multiplicação em potes de cultura para propiciar maior esporulação dos FMAs.

- A mineração de gesso reduz a diversidade de espécies de FMAs em áreas de caatinga.

- A similaridade de espécies de FMAs entre áreas impactadas no mesmo ecossistema decorre, possivelmente, da ocorrência de espécies adaptadas ao estresse ambiental.

Estudos no campo devem ser ampliados para melhor compreensão da dinâmica e funcionalidade de Glomus intraradices e Paraglomus occultum, espécies mais registradas nas áreas impactadas, abrindo a possibilidade de seleção desses fungos para aplicação em programa de manejo de áreas alteradas por mineração de gesso.

\section{AGRADECIMENTOS}

Aos revisores anônimos, pelas valiosas modificações sugeridas.

R. Árvore, Viçosa-MG, v.33, n.2, p.227-236, 2009

\section{REFERÊNCIAS}

ALLEN, E. et al.. Patterns and regulation of mycorrhizal plant and fungal diversity. Plant and Soil, v.170, n.1, p.47-62, 1995.

BROWER, J. E.; ZAR, J. H.. Community similarity. In: BROWER, J. E.; ZAR, J. H.; VON ENDE, C. N. Field \& laboratory methods for general ecology. 2.ed. Dubuque: W M C. Brown Publishers, 1984. 226p.

BONONI, V. L. R.; TRUFEM, S. F. B.

Endomicorrizas vesículo-arbusculares do cerrado da Reserva Biológica de Moji-Guaçú, Brasil.

Rickia, v.10, n.1, p.55-84, 1983.

CAPRONI, A. L. et al. FMAs em estéril revegetado com Acacia mangium, após mineração de bauxita. Revista Árvore, v.29, n.3, p.373-381, 2005.

CAPRONI, A. L. et al. Capacidade infectiva de fungos micorrízicos arbusculares em áreas reflorestadas após mineração de bauxita no Pará. Pesquisa Agropecuária Brasileira v.38, n.12, p.937-945, 2003.

CARRENHO, R.; TRUFEM, S. F. B.; BONONI, V.L. Effects of using different host plants on the detected biodiversity of arbuscular mycorrhizal fungi from an agroecosystem. Revista Brasileira de Botânica, v.25, n.1, p.93-101, 2002.

CAVAlCANTI, A. C.; LOPES, O. F. Condições edafoclimáticas da Chapada do Araripe e viabilidade de produção sustentável de culturas. Brasília: Embrapa, 1994. 42p.

D'ARCY-LAMETA, A. Study of soybean and lentil root exudates. II. Identification of some polyphenolic compounds, related with plant physiology. Plant and Soil, v.92, n.1, p.113-123, 1988.

FITTER, A. H. The role and ecological significance of vesicular-arbuscular mycorrhizas intemperate ecosystems. Agricultural Ecosystems Environmental, v.29, n.1, p.137-151, 1990.

GERDEMANN, J. W.; NICOLSON, T. H. Spore of mycorrhizal Endogone species extracted from soil by wet sieving and decanting. Transactions of the British Mycological Society, v.46, n.2, p.235-244, 1963. 
GIANINAZZI-PEARSON, V.; BRANZATI, V.; GIANINAZZI, S.. In vitro enhancement of spore germination and early hyphal growth of a vesicular-arbuscular mycorrhizal fungus by host root exudates and plant flavonoids. Symbiosis, v.7, n.3, p.243-255, 1989

GUSMAO, L. P.; MAIA, L. C. (Orgs.). Diversidade e caracterização dos fungos do Semi-árido Brasileiro. Recife, Associação Plantas do NordesteAPNE, 2006.

JENKINS, W. R. A rapid centrifugal-flotation technique for separating nematodes from soil. Plant Disease Repórter, v.48, p.692, 1964.

JESUS, E. C.; SCHIAVO, J. A.; FARIAS, S. M. Dependência de micorrizas para a nodulação de leguminosas arbóreas tropicais. Revista Árvore, v.29, n.4, p.545-552, 2005.

MARINHO, N. F. et al. Respostas de Acacia mangium Willd e Sclerolobium paniculatum Vogel a fungos micorrízicos arbusculares nativos provenientes de áreas degradadas 2004.pela mineração de bauxita na Amazônia. Acta Botanica Brasílica, v. 18, n.1, p.141-149, 2004.

MELLONI, R.; SIQUEIRA, J. O.; MOREIRA, F. M. S. Fungos micorrízicos arbusculares em solos de área de mineração de bauxita em reabilitação. Pesquisa Agropecuária Brasileira, v.38, n.2, p.267-276, 2003.

MERGUlhão, A. C. E. S. et al. Potencial de infectividade de fungos micorrízicos arbusculares em áreas nativas e impactadas por mineração gesseira no semi-árido brasileiro. Hoehnea, v.34, n.3, p.341-348, 2007.

OEHL, F. et al. Impact of long-term conventional and organic farming on the diversity of arbuscular mycorrhizal fungi. Oecologia, v.138, n.4, p.574-583, 2004.

PETERS, N. K.; FROST, J. W.; LONG, S. R. A plant flavone luteolin induces expression of Rhizobium meliloti nodulation genes. Science, v.233, n.1, p.77-99, 1986.
PHILLIPS, J. M.; HAYMAN, D. S. Improved produces for clearing roots and staining parasitic and vesicular-arbuscular micorrhizal fungi for rapid assement of infection. Transactions of the British Mycological Society, v.55, p.158-161, 1970.

REDMOND, J. W. et al. Flavones induce expression of nodulating genes in Rhizobium. Nature, v.323, p.632-635, 1986.

REEVES, F. B.; REDENTE, E. F. The importance of mutualism in sucession. In: SKUJINS, J. (Ed.). Semiarid lands and deserts, soil resource and reclamation New York: Marcel Dekker, 1991. p.423-442.

ROBERTSON, W. K. et al. Effects of plant water stress on root distribution of corn, soybeans and peanuts in a sandy soil. Agronomy Journal, v.72, p.548-550, 1980.

SCHENCK, N. C.; PÉREZ, Y. Manual for the identification of VA mycorrhizal fungi. 3.ed. Gainesville, Synergistic Publication, 1990.

SIEVERDING, E. Vesicular-arbuscular mycorrhiza management in tropical agrosystems. Eschborn: Deutsche Gesellschaft fü Technische Zusammenarbeit (GTZ) 1991.

SILVA, G. A. et al. Potencial de infectividade de fungos micorrízicos arbusculares oriundos de área de caatinga nativa e degradada por mineração, no Estado da Bahia, Brasil. Revista Brasileira de Botânica, v.24, n.1, p.135-143, 2001.

SILVA, G. A. et al. Arbuscular mycorrhizal fungi in a semiarid copper mining área in Brazil.

Mycorrhiza, v.15: n.1, p.47-53, 2005.

SIQUEIRA, J. O.; KLAUBERG FILHO, O. Micorrizas arbusculares: a pesquisa brasileira em perspectiva. In: NOVAIS, R. F.; ALVAREZ V., V. H.; SCHAEFER, C. E. G. R. (Eds.). Tópicos em ciência do solo. Viçosa-MG: Sociedade Brasileira de Ciência do Solo, 2000. p.235-259.

SOUZA, F. A.; SILVA, E. M. R. Micorrizas arbusculares na revegetação de áreas degradadas.. In: SIQUEIRA, J. 0. (Ed). Avanços em fundamentos e aplicação de micorrizas. Lavras: UFLA/DCS e DCF, 1996. p.255-290.

R. Árvore, Viçosa-MG, v.33, n.2, p.227-236, 2009 
SOUZA, R. G. et al. Diversidade e potencial de infectividade de fungos micorrízicos arbusculares em áreas de caatinga, na região de Xingó, Estado de Alagoas, Brasil. Revista Brasileira de Botânica, v.26, n.1, p.49-60, 2003.

STUTZ, J. C.; MORTON, J. B. Sucessive pot cultures reveal high species richness of arbuscular endomycorrhizal fungi in arid ecosystems. Canadian Journal of Botany, v.74, p.1883-1889, 1996.

TOMÉ JÚNIOR, J. B. Interpretação dos resultados.. In: TOMÉ JÚNIOR, J. B. (Ed). Manual para interpretação de análise do solo. Guaíba: Agropecuária, 1997. p.89-107.
VAN DER HEIDJEN, M. G. A. et al. Different arbuscular mycorrhizal fungal species are potential determinants of plant community structure. Ecology, v.79, n.6, p.2082-2091. 1998.

YANO-MELO, A. M.; TRUFEM, S. F. B.; MAIA, L. C. Arbuscular mycorrhizal fungi in salinized and surrounded areas at the São Francisco Submedium Valley, Brazil.

Hoehnea, v.30, n.2, p.79-87, 2003.

ZONTA, E. P.; MACHADO, A. A.; SILVEIRA JÚNIOR, P. Sistema de análise estatística para microcomputadores (SANEST).

Pelotas: Departamento de Matemática e Estatística, 1984. 